\title{
Comparison of turbulence models and CFD solution options for a plain pipe
}

\author{
Eyub Canli ${ }^{1, *}$, Ali Ates ${ }^{1}$, and Sefik Bilir $^{1}$ \\ ${ }^{1}$ Selcuk University, Mechanical Engineering Department, P.K. 42003 Selcuklu Konya, Turkey
}

\begin{abstract}
Present paper is partly a declaration of state of a currently ongoing $\mathrm{PhD}$ work about turbulent flow in a thick walled pipe in order to analyze conjugate heat transfer. An ongoing effort on CFD investigation of this problem using cylindrical coordinates and dimensionless governing equations is identified alongside a literature review. The mentioned $\mathrm{PhD}$ work will be conducted using an in-house developed code. However it needs preliminary evaluation by means of commercial codes available in the field. Accordingly ANSYS CFD was utilized in order to evaluate mesh structure needs and asses the turbulence models and solution options in terms of computational power versus difference signification. Present work contains a literature survey, an arrangement of governing equations of the PhD work, CFD essentials of the preliminary analysis and findings about the mesh structure and solution options. Mesh element number was changed between 5,000 and 320,000. $k-\epsilon, k-\omega$, Spalart-Allmaras and Viscous-Laminar models were compared. Reynolds number was changed between 1,000 and 50,000. As it may be expected due to the literature, $k-\epsilon$ yields more favorable results near the pipe axis and $k-\omega$ yields more convenient results near the wall. However $k-\epsilon$ is found sufficient to give turbulent structures for a conjugate heat transfer problem in a thick walled plain pipe.
\end{abstract}

\section{Introduction}

Flow inside a thick walled pipe and related heat transfer is of interest. Numerical and computational means can be used for investing a wide range of mentioned interest and compact results can be reported in a way that the results can be converted and applied to a wide application variety and field. The compact results can be derived by scaling and using dimensionless governing equations.

A dedicated team in Turkey has been performing numerical studies on unsteady and steady conjugate heat transfer related to thick walled pipes under various conditions such as periodic boundary conditions, simultaneously developing flow, rarefaction effects and slippery flow, heat transfer in tangential direction and etc by using cylindrical coordinates and their own developed codes. As mentioned earlier, the results are dimensionless and can be expanded for certain magnitudes. Remarkable developments and major events are summarized below according to the publishing timeline.

After a detailed and informative $\mathrm{PhD}$ dissertation was published in 1988 [1] about heat transfer in laminar pipe flow in the thermal development region at low Peclet numbers including axial conduction, Bilir (1995) reported a numerical laminar heat transfer analysis including two dimensional axial conduction for the wall and for the fluid [2]. The pipe in the problem was constituted by two regions and second region causes a step change in outside surface temperature. This causes heat transfer through the wall and the fluid. Peclet number, thickness ratio and wall-to-fluid conductivity ratio were taken as the investigated parameters while Peclet number was found as the most effective parameter among them. It should also worth that the numerical code of [1] is also given in it. Bilir then reported a solution for transient regime of the previously mentioned problem [3]. This time parameters were increased as wall-to-fluid thermal diffusivity ratio was added into the analysis. As a main conclusion of the work, author reported that the time passed in order to reach the steady state conditions does not change significantly with the parameters although the early and intermediate periods exhibit differences. It was also stressed that flow conditions determine the thermal inertia rather than the wall specifications.

In 2003, a different boundary condition was reported by Bilir and Ates [4]. Convective boundary condition was applied to downstream region of the numerical domain which was consist of two regions. Also Biot number was taken as an additional parameter which makes the total parameter number as five. Considerable amount of axial conduction towards upstream was reported. The effect of parameters on the time to reach steady state condition was found to be significant. Also it is given that there are certain values of parameter after which results are not changing significantly. The details also can be found in the dissertation [5]. Problem then modified in respect of the parameters and results are

Corresponding author: ecanli@selcuk.edu.tr 
reported [6]. Their main findings can be summarized as the preheating phenomenon due to the axial heat transfer towards the upstream; reverse heat flow (from fluid to wall) at the early transient stages in upstream; peak values at the interface of upstream and downstream due to the surpassing radial wall conduction; and axial heat conduction towards downstream region through the wall in steady state conditions making the heat flux values at fluid wall interface greater than the outer surface where heat flux boundary condition exists.

First time numerical solution of the flow of the team was in 2004 where laminar flow development was obtained for simultaneously developing flow [7]. The effect of the velocity profile other than the parabolic velocity profile is investigated and results are given comparatively in terms of the outer and inner wall temperatures, bulk temperatures, radial distributions of the fluid temperatures and Nusselt numbers [8]. It is said that velocity profile has no effect on interface and outer surface temperatures. Bulk temperature values on the other hand are higher for developing flow comparing to the developed flow. Slug flow for the thick walled pipe is also examined numerically by the team [9]. For this flow type, temperature profiles develop faster in the order of outer surface, interface and bulk flow. They validated their results by considering the Nusselt number for developed flow with constant temperature condition. Since thick walled pipes can be seen in applications involving micro-channels and mini-pipes frequently, the parameters were arranged by the authors and reported [10]. There are six parameters which are wall thickness ratio, wall-to-fluid thermal conductivity ratio, wall-tofluid thermal diffusivity ratio, the Peclet number, the Biot number and the Prandtl number. It is stated that the interfacial heat flux rises rapidly at initiation of the transient regime due to fast radial conduction in the wall at the entrance of the pipe. Then it reaches a constant value in a short axial distance. Again it is noticed that heat flux values decrease in the mean and uniformity of the curves are deformed as time elapses because of the increasing effect of convection in the fluid side and after a maximum they decrease in the flow direction. As an analogy to electrical current, conjugation increases with the diameter. Remaining parameters reduce it. The critic values of the parameters are also given and after these critic values towards a certain direction, results don't change significantly. Only Pr number doesn't affect time to reach steady state conditions significantly.

Periodic boundary conditions for the thick walled pipe in which heat conjugation takes place for laminar flow was also dealt numerically by Altun [11]. A paper was also published on periodic outer wall temperature changing with time [12]. It can be inferred from the results that the periodicity can be observed in the plots identically for all parameter values while parameters change the peak values. The additional parameter of the work is the angular frequency. Demirpolat [13] repeated the analysis for periodic convective boundary conditions.

Heat transfer in tangential direction is taken into consideration in the dissertation of Atmaca [14]. Transient conjugated heat transfer in circumferentially half heated thick walled pipes for thermally developing laminar flows is investigated in the study. As expected, an intensive heat transfer occurs towards the insulated part of the pipe by tangential conduction.

Finally Sen completed a $\mathrm{PhD}$ thesis on slippery flow which can be attributed to gas flow in micro channels [15]. In its paper [16], it is stated that momentum and energy equations are solved with the boundary conditions of the first degree velocity slip and temperature jump. The independent parameters are Peclet number, the Knudsen number, the Brinkman number and the wall thickness ratio. Their major conclusion can be summarized as; fluid axial conduction is effective in the entrance region while it can be neglected in the remaining part; Interfacial heat flux values decrease with increasing Knudsen numbers; reverse heat flow occurs in upstream region with increase in Brinkman number; and thermal development length increases with increasing diameter and decreasing Peclet number.

Currently a $\mathrm{PhD}$ thesis (of the first author) is going on by the team in order to investigate the heat transfer for a turbulent flow in a thick walled pipe numerically by using finite elements. This should be considered a further step of the accumulated knowledge. Literature is also surveyed for similar cases.

The deterministic near-wall turbulence model was used by Kasagi et al. for calculation and simulation of near wall turbulent heat transfer involving the effect of unsteady heat conduction in the solid wall [17]. They introduced three fluctuating velocity components into the governing equations not by using the Reynolds decomposition method but using algebraic expressions along with their approach. Temperature variance, turbulent heat flux, turbulent Prandtl number and some more statistical data are reported for fluids with different Prandtl numbers. They stated that the wall thickness and thermal properties affect the near wall values.

Cao and Faghri studied turbulent forced convection of a transport fluid by using $k-\epsilon$ model and coupling it with the phase change solution because the application of interest is a phase changing energy storage application [18]. They validated their code with different experimental data and then used the code for other conjugated heat transfer scenarios. A correlation for the energy storage capacity was derived and its empirical constants were determined by the numerical data and presented.

Jaeger investigated the heat transfer to liquid metals for forced convection in various geometries by using empirical models [19]. Since liquid metals are mentioned here, one can think that laminar flow should be considered here. The author also emphasized that this fact. The empirical formulas in order to represent thermal-hydraulic behavior of several liquid metals in the numerical codes were compiled and used. This work can be regarded as a validation reference where liquid metals or specific conditions that can be attributed to the liquid metals are of interest.

A direct numerical solution of turbulent heat transfer in a channel while wall conduction exists was reported by Tiselj et al. [20]. They investigated unsteady conduction in the heated walls for fully developed flow 
conditions. The flow field is two dimensional. They proposed that it can be decided, which behavior has to be expected in a real fluid-solid system and which one of the limiting boundary conditions is valid for calculation, or whether more expensive conjugate heat transfer calculation is required. They also stated the weakness of the previous studies as underestimating the wall temperature fluctuations for the limiting cases of the ideal-isoflux boundary conditions.

DNS of turbulent channel flow with conjugate heat transfer at Prandtl number 0.01 is performed by Tiselj and Cizelj [21]. This Pr number practically corresponds to the liquid sodium. They used pseudo-spectral channelflow code. As a summary of their findings, it can be said that the log law region cannot be observed by the temperature profile of the fluid, even for the highest implemented Reynolds number; the near-wall RMS temperature fluctuations show Reynolds number dependence; an intensive turbulent temperature fluctuation penetrates towards the wall according to the conjugate heat transfer simulations.

In the subsequent sections, governing equations of the quoted study and supplementary information are given. Also the design of the ANSYS CFD preliminary analysis is presented. Turbulent flow is calculated at the entrance region of a pipe by means of four viscous flow model using ANSYS CFD. Results are given comparatively in terms of $\mathrm{u}$ velocity profiles versus coordinate axes, wall $\mathrm{y}^{+}$, turbulence viscosity and intensity values.

\section{Method}

Governing equations of a case involving numerical investigation of a turbulent flow and heat transfer for incompressible constant viscosity flow can be given in vector form are listed below between (1-3) [22]:

$$
\begin{gathered}
\frac{\partial \rho}{\partial t}+\nabla \cdot(\rho \mathbf{V})=0 \\
\rho \frac{D \mathbf{V}}{D t}=\mathbf{f}-\nabla p+\mu \nabla^{2} \mathbf{V} \\
\rho \frac{\partial e}{\partial t}+p(\nabla \mathbf{V})=\nabla(k \nabla T)+\Phi
\end{gathered}
$$

(1) is continuity, (2) is the momentum equation and (3) is the energy equation. The extended forms of (1-3) for two dimensional steady flow in cylindrical coordinate system after Reynolds decomposition and neglecting viscous dissipation are given below $[9,22]$ :

$$
\begin{gathered}
\frac{\partial \bar{u}}{\partial x}+\frac{\bar{v}}{r}+\frac{\partial \bar{v}}{\partial r}=0 \\
\bar{u} \frac{\partial \bar{u}}{\partial x}+\bar{v} \frac{\partial \bar{u}}{\partial r}=-\frac{1}{\rho} \frac{\partial p}{\partial x}+v\left[\frac{1}{r} \frac{\partial}{\partial r}\left(r \frac{\partial \bar{u}}{\partial r}\right)+\frac{\partial^{2} \bar{u}}{\partial x^{2}}\right] \\
+\frac{1}{r} \frac{\partial}{\partial r}(-r \vec{v} \vec{u})+\frac{\partial}{\partial x}\left(-\overrightarrow{u^{\prime} u}\right)
\end{gathered}
$$

$$
\begin{aligned}
& \bar{u} \frac{\partial \bar{v}}{\partial x}+\bar{v} \frac{\partial \bar{v}}{\partial r}=-\frac{1}{\rho} \frac{\partial p}{\partial r}+v\left[\frac{\partial}{\partial r}\left(\frac{1}{r} \frac{\partial(r \bar{v})}{\partial r}\right)+\frac{\partial^{2} \bar{v}}{\partial x^{2}}\right] \\
& +\frac{1}{r} \frac{\partial}{\partial r}\left(-r \overrightarrow{v^{\prime} v}\right)+\frac{\partial}{\partial x}\left(-\overrightarrow{u^{\prime} v^{\prime}}\right) \\
& \bar{u} \frac{\partial \bar{T}}{\partial x}+\bar{v} \frac{\partial \bar{T}}{\partial r}=\alpha\left[\frac{\partial}{\partial x}\left(\frac{\partial \bar{T}}{\partial x}\right)+\frac{1}{r} \frac{\partial}{\partial r}\left(r \frac{\partial \bar{T}}{\partial r}\right)\right] \\
& +\frac{\partial}{\partial x}\left(-\overrightarrow{u^{\prime} T^{\prime}}\right)+\frac{1}{r} \frac{\partial}{\partial r}\left(-r \overrightarrow{v^{\prime} T^{\prime}}\right)
\end{aligned}
$$

Fluctuating velocity component and temperature values take place in the equations as it can be seen from (4-7). These averaged products will be modeled by $k-\epsilon$ while they can be given as;

$$
\begin{gathered}
-\overrightarrow{u_{i}^{\prime} u_{j}^{\prime}}=v_{T}\left(\frac{\partial \bar{u}_{i}}{\partial x_{j}}+\frac{\partial \bar{u}_{j}}{\partial x_{i}}\right)-\frac{2}{3} k \delta_{i j} \\
-\overrightarrow{v^{\prime} u}=\overrightarrow{u^{\prime} v}=v_{T}\left(\frac{1}{r} \frac{\partial r \bar{u}}{\partial r}+\frac{\partial \bar{v}}{\partial x}\right) \\
-\overrightarrow{u^{\prime} u}=v_{T}\left(2 \frac{\partial \bar{u}}{\partial x}\right)-k \\
-\overrightarrow{v^{\prime} v}=v_{T}\left(\frac{2}{r} \frac{\partial r \bar{v}}{\partial r}\right)-k \\
-\overline{u^{\prime} T}=\alpha_{T} \frac{\partial \bar{T}}{\partial x} \\
-\overrightarrow{v^{\prime} T}=\alpha_{T} \frac{1}{r} \frac{\partial \bar{T}}{\partial r} \\
k=\frac{1}{2}\left(\overline{u^{\prime 2}}+\overline{v^{\prime 2}}\right)
\end{gathered}
$$

Here $v_{T}$ is the turbulence or eddy viscosity and it is not a property of the fluid. Instead it is a property of the flow and it changes according to the location and can be related to the average flow properties. Kronecker delta $\delta_{i j}$ enables to consider situations only for $i=j$. When $i=j$ the first parenthesis of right hand side of the (8) would yield a normal stress and the addition of the two normal stresses would be zero due to the continuity. However all normal stresses are positive by their definition. Also it is known that the sum of normal stresses gives the kinetic energy of the turbulent flow which is multiplied by two. The second part of (8) ensures that the sum of the normal stresses is not zero. $k$ then can be absorbed into the pressure when inserting turbulence viscosity into the governing equations and therefore it is not necessary to calculate $k$. Instead, $v_{T}$ distribution is tried to be obtained. In the $k-\epsilon, v_{T}$ is modeled by a velocity scale $(\sqrt{k})$ and a length scale $\left(k^{3 / 2} / \varepsilon\right)$. (15) is given as transport equation of $k$ and (16) is given as the transport equation of $\epsilon[23,24]$. 


$$
\begin{gathered}
\frac{\partial k}{\partial t}+\bar{u}_{i} \frac{\partial k}{\partial x_{i}}=\frac{\partial}{\partial x_{i}}\left(\frac{v_{T}}{\sigma_{k}} \frac{\partial k}{\partial x_{i}}\right)+G-\epsilon \\
\frac{\partial \epsilon}{\partial t}+\bar{u}_{i} \frac{\partial \epsilon}{\partial x_{i}}=\frac{\partial}{\partial x_{i}}\left(\frac{v_{T}}{\sigma_{\epsilon}} \frac{\partial \epsilon}{\partial x_{i}}\right)+C_{1 \epsilon} \frac{\epsilon}{k} G-C_{2 \epsilon} \frac{\epsilon^{2}}{k}
\end{gathered}
$$

Here G (17) is the generation term and represent the generation of $\mathrm{k} . k$ and $\epsilon$ can be related to each other as in (18). The coefficients appear in the equations can be viewed in (19).

$$
\begin{gathered}
G=v_{T}\left(\frac{\partial \bar{u}_{i}}{\partial x_{j}}+\frac{\partial \bar{u}_{j}}{\partial x_{i}}\right) \frac{\partial \bar{u}_{i}}{\partial x_{j}} \\
v_{T}=C_{\mu} \frac{k^{2}}{\epsilon} \\
C_{\mu}=0.09, C_{1 \epsilon}=1.44, C_{2 \epsilon}=1.92, \sigma_{k}=1.0, \sigma_{\epsilon}=1.3
\end{gathered}
$$

When the equations are put in an order and reorganized for $2 \mathrm{D}$ steady flow in cylindrical coordinates; a similar approach to above can be applied for the energy equation. The turbulence viscosity can be related to the turbulence diffusion coefficient as in (23).

$$
\begin{gathered}
G=v_{t}\left[2\left(\frac{\partial \bar{u}}{\partial x}\right)^{2}+2\left(\frac{1}{r} \frac{\partial r \bar{v}}{\partial r}\right)^{2}+\left(\frac{1}{r} \frac{\partial r \bar{u}}{\partial r}+\frac{\partial \bar{v}}{\partial x}\right)^{2}\right. \\
\bar{u} \frac{\partial k}{\partial x}+\bar{v} \frac{\partial k}{\partial r}=\frac{\partial}{\partial x}\left(\frac{v_{T}}{\sigma_{k}} \frac{\partial k}{\partial x}\right)+\frac{1}{r} \frac{\partial}{\partial r}\left(\frac{v_{T}}{r \sigma_{k}} \frac{\partial r k}{\partial r}\right)+G-\epsilon \\
\bar{u} \frac{\partial \epsilon}{\partial x}+\bar{v} \frac{\partial \epsilon}{\partial r}=\frac{\partial}{\partial x}\left(\frac{v_{T}}{\sigma_{\epsilon}} \frac{\partial \epsilon}{\partial x}\right)+\frac{1}{r} \frac{\partial}{\partial r}\left(\frac{v_{T}}{r \sigma_{\epsilon}} \frac{\partial r \epsilon}{\partial r}\right) \\
+C_{1 \epsilon} \frac{\epsilon}{k} G-C_{2 \epsilon} \frac{\epsilon^{2}}{k} \\
\alpha_{T}=\frac{v_{T}}{\sigma_{T}}
\end{gathered}
$$

$\sigma_{T}$ is named as turbulence Prandtl number or Schmidt number and it was shown that its value is nearly constant by means of experiments. 0.9 is reported as an appropriate value. However it is also stated that the coefficient is affected by buoyancy and streamline curvature. If the RANS equations are re-written by using turbulence viscosity and turbulence diffusion coefficient for $k-\epsilon$ model;

$$
\begin{gathered}
\bar{u} \frac{\partial \bar{u}}{\partial x}+\bar{v} \frac{\partial \bar{u}}{\partial r}=-\frac{1}{\rho} \frac{\partial p}{\partial x}+v\left[\frac{1}{r} \frac{\partial}{\partial r}\left(r \frac{\partial \bar{u}}{\partial r}\right)+\frac{\partial^{2} \bar{u}}{\partial x^{2}}\right] . \\
+\frac{1}{r} \frac{\partial}{\partial r}\left(r v_{T}\left(\frac{1}{r} \frac{\partial r \bar{u}}{\partial r}+\frac{\partial \bar{v}}{\partial x}\right)\right)+\frac{\partial}{\partial x}\left(v_{T}\left(2 \frac{\partial \bar{u}}{\partial x}\right)-k\right) \\
\bar{u} \frac{\partial \bar{v}}{\partial x}+\bar{v} \frac{\partial \bar{v}}{\partial r}=-\frac{1}{\rho} \frac{\partial p}{\partial r}+v\left[\frac{\partial}{\partial r}\left(\frac{1}{r} \frac{\partial(r \bar{v})}{\partial r}\right)+\frac{\partial^{2} \bar{v}}{\partial x^{2}}\right]
\end{gathered}
$$

$$
\begin{array}{r}
+\frac{1}{r} \frac{\partial}{\partial r}\left(r v_{T}\left(\frac{2}{r} \frac{\partial r \bar{v}}{\partial r}\right)-k\right)+\frac{\partial}{\partial x}\left(v_{T}\left(\frac{1}{r} \frac{\partial r \bar{u}}{\partial r}+\frac{\partial \bar{v}}{\partial x}\right)\right) \\
\bar{u} \frac{\partial \bar{T}}{\partial x}+\bar{v} \frac{\partial \bar{T}}{\partial r}=\frac{\partial}{\partial x}\left[\left(\alpha+\alpha_{T}\right) \frac{\partial \bar{T}}{\partial x}\right]+\frac{1}{r} \frac{\partial}{\partial r}\left[\left(\alpha r+\alpha_{T}\right) \frac{\partial \bar{T}}{\partial r}\right]
\end{array}
$$

As summarized above, this work will be conducted by an in-house developed code. In order to narrow the starting range of investigation and validate results comparatively, a series of preliminary analyses is targeted by utilizing commercial codes and/along with open sourced codes. As a first step, present paper contains CFD results of a case using Ansys CFD. Details are given below.

The Ansys CFD analysis is two dimensional and axisymmetric. So the flow domain was drawn as a rectangle after the settings of Ansys Geometry was set to "2D". A schematic drawing of the geometry is given in Fig. 1.

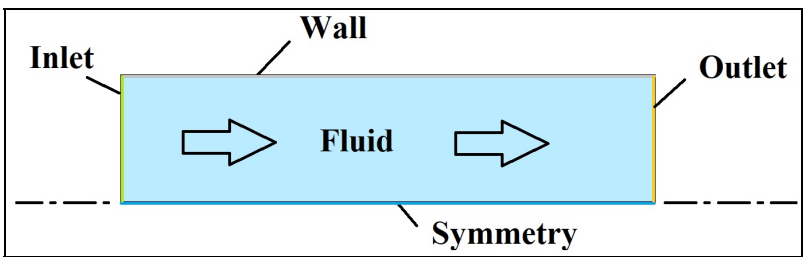

Fig. 1. Schematic view of the geometry and flow domain

The grid (mesh) was constituted by 20,000 (50x400) quadrilateral cells. 50 cells were located at the inlet along the radius and 400 cells were located at the symmetry along the symmetry axis. A bias was applied to all edges of the geometry by "edge sizing" option in a way that the cell number for an edge stays constant but the edge length of the cell on the edge of the geometry stretches towards symmetry axis and outlet by the bias factor of 10. A "face mesh" option was also applied to have a structured mesh. The node number of the mesh is 20,451. Maximum mesh skeweness is $1.31 \times 10^{-10}$ which can be viewed a success of the structural mesh. The view of the inlet and outlet parts of the mesh is given in Fig.3. In order to asses if the mesh is adequate for the solution, the element number at $\mathrm{x}$ and $\mathrm{y}$ axes are doubled and halved without touching the bias factors and face meshing of the structural mesh. At this point, 5,000, 80,000 and 320,000 elements were tried. Table 1 shows five different results at the outlet of the pipe for four different element number values. Figure 2 also given for a comparison of different element numbers. According to the results, 80,000 element number was chosen for the comparison of the viscous flow models since tradeoff between time cost and change in turbulent values is satisfactory.

Table 1. Grid comparison $-k-\epsilon \operatorname{Re}=10,000$ at pipe outlet

\begin{tabular}{|c|c|c|c|c|}
\cline { 2 - 5 } \multicolumn{1}{c|}{5,000} & 20,000 & 80,000 & 320,000 \\
\hline Vel. (m/s) & 0.1219 & $0.1091(10 \%)$ & $0.1041(4.5 \%)$ & $0.0942(9.5 \%)$ \\
\hline Tot. Pres. (Pa) & 7.45 & $5.95(20 \%)$ & $5.42(9 \%)$ & $4.44(18 \%)$ \\
\hline Turb. Int. & 0.1362 & $0.3494(156 \%)$ & $0.443(26 \%)$ & $0.4385(1 \%)$ \\
\hline $\begin{array}{c}\text { Turb. Vis. } \\
\text { (Pa*) }\end{array}$ & 0.0008 & $0.019(2387 \%)$ & $0.0265(33 \%)$ & $0.0215(18 \%)$ \\
\hline y+ & 2.049 & $1.058(48 \%)$ & $0.587(44 \%)$ & $0.349(40 \%)$ \\
\hline Av. \% Dif. & - & $524.2 \%$ & $23.3 \%$ & $17.3 \%$ \\
\hline
\end{tabular}



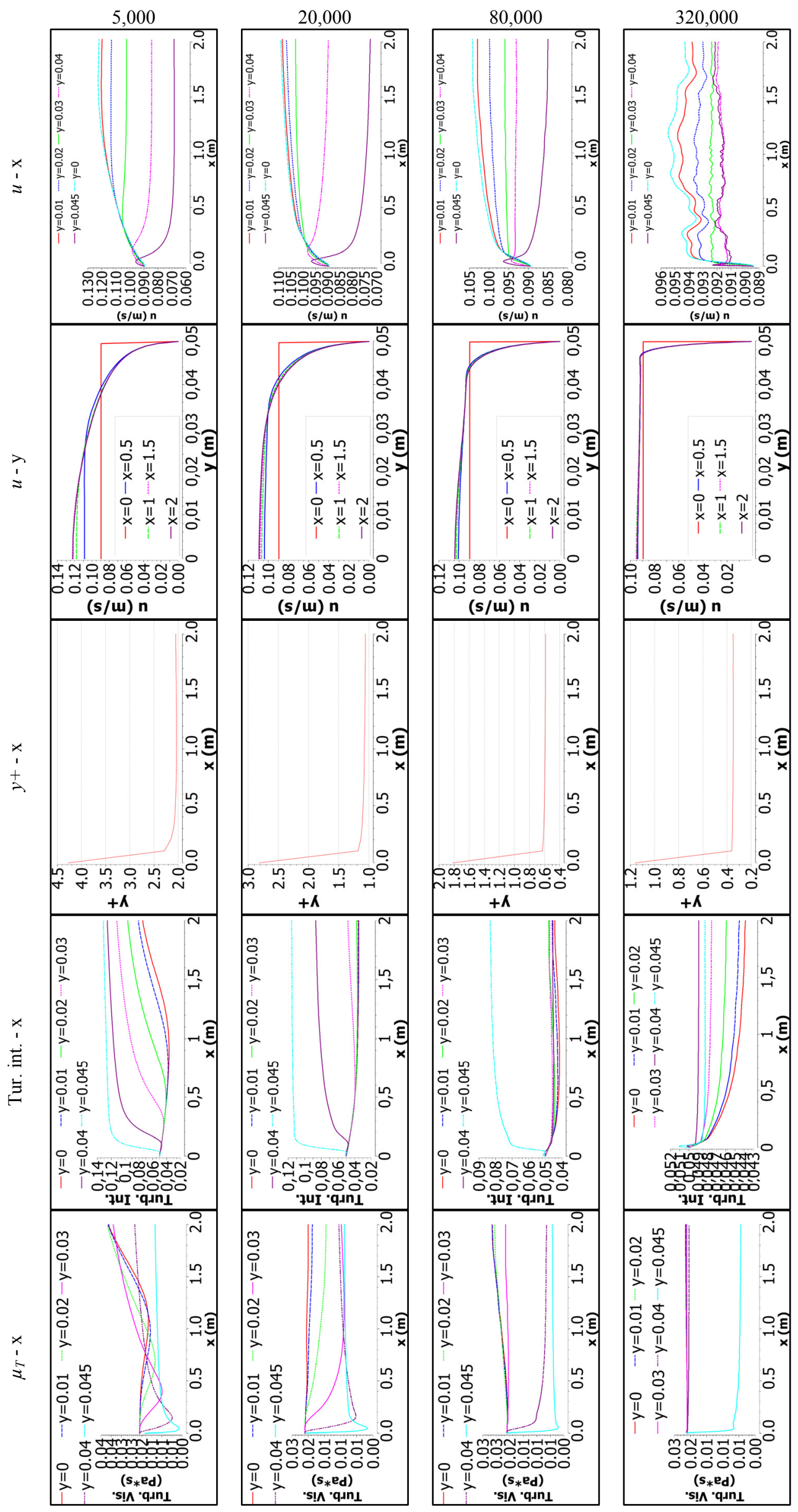

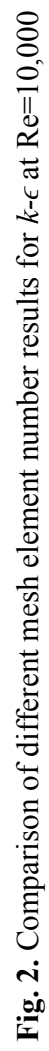


Although element number changes for the grid independency, Figure 3 gives an idea about the structural mesh and the bias factor.

Four viscous flow models were used; Viscous Laminar, Spalart-Allmaras, $k-\epsilon$ and $k-\omega$. Then the software was run for four different Re numbers; 50,000, $10,000,5,000$ and 1,000 for the selected turbulence model. Five different two dimensional plot were drawn after the computations where it was possible; u velocity versus $\mathrm{x}$ axis (or pipe length), $\mathrm{u}$ velocity versus $\mathrm{y}$ axis (or pipe radius), wall $\mathrm{y}+$ versus $\mathrm{x}$ axis, turbulent viscosity versus $\mathrm{x}$ axis and turbulent intensity versus $\mathrm{x}$ axis. These two dimensional plots were drawn for different line positions; for instance, lines starting at different " $y$ " axis points at the inlet lie towards the outlet parallel to the wall and the symmetry axis were used for the profiles.

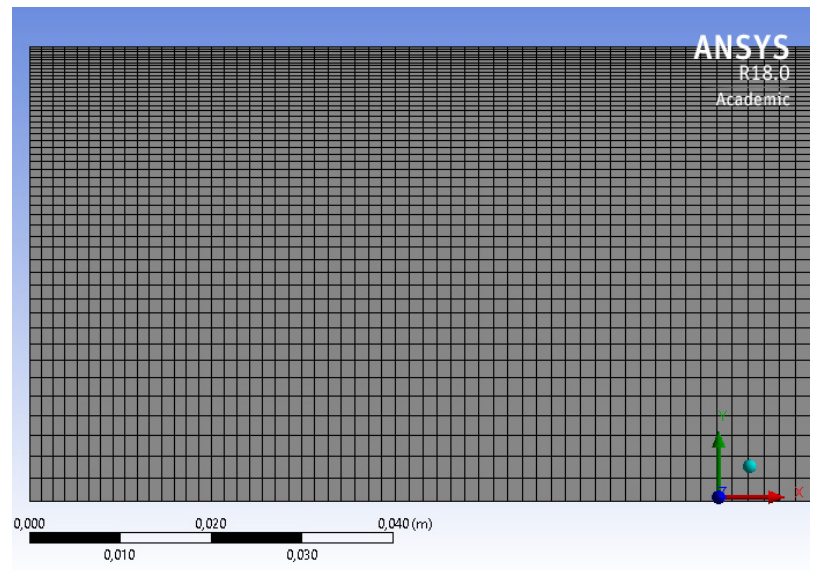

(a)

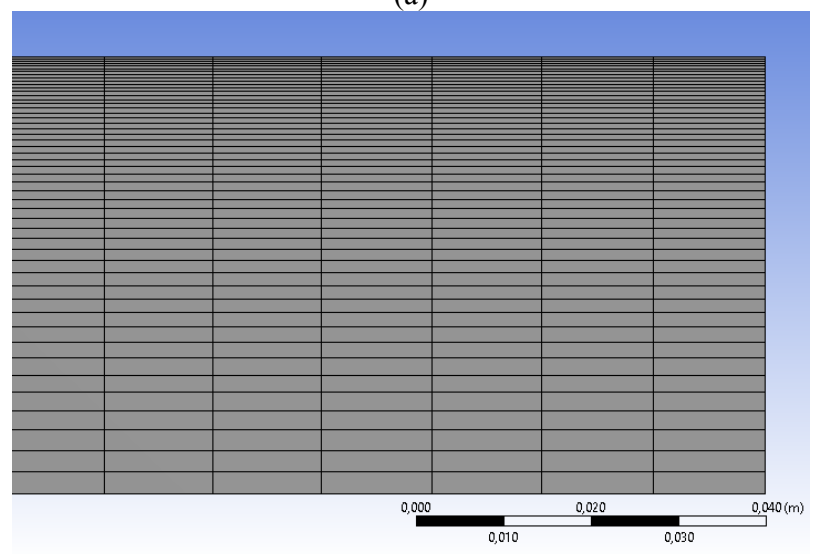

(b)

Fig.3. (a) mesh view at inlet (b) mesh view at outlet

Fluent solver of the Ansys CFD ran for viscous flow models. General setup can be summarized as; 2D, single precision, serial processing, pressure based, axisymmetric and without gravity. Inlet boundary condition was selected as constant velocity in a way that is ensuring the desired Re number according to the pipe diameter. Outlet boundary condition was set to pressure outlet and its value is fixed as $0 \mathrm{~Pa}$ gauge pressure. Hybrid initialization was used. Iteration numbers change between 29 and 145. Solution converges in seconds (between 5 and 30 seconds) since 320,000 elements only used once for the grid examination and mainly 80,000 elements were used. Transition Re number (i.e. 5,000 in this case) caused highest iteration numbers except for $k$ $\epsilon$. The highest iteration number was 145 for $k$ - $\epsilon$ and $\mathrm{Re}=1,000$. The least iteration number was 88 for $k-\epsilon$ and $\mathrm{Re}=50,000$. 0.001 was selected as the convergence criteria for all residuals. $\epsilon$ was the last converged magnitude among $k$, continuity and velocity components for $k-\epsilon$. $\omega$ was the first to converge for $k-\omega$. "y" velocity was converge in the order of first two for all models as expected. Most of the time for the analysis was spent for structural mesh and reporting.

CFD-Post was used for the post processing. The 3D view of the $2 \mathrm{D}$ flow domain in CFD-Post is given in Figure 4.

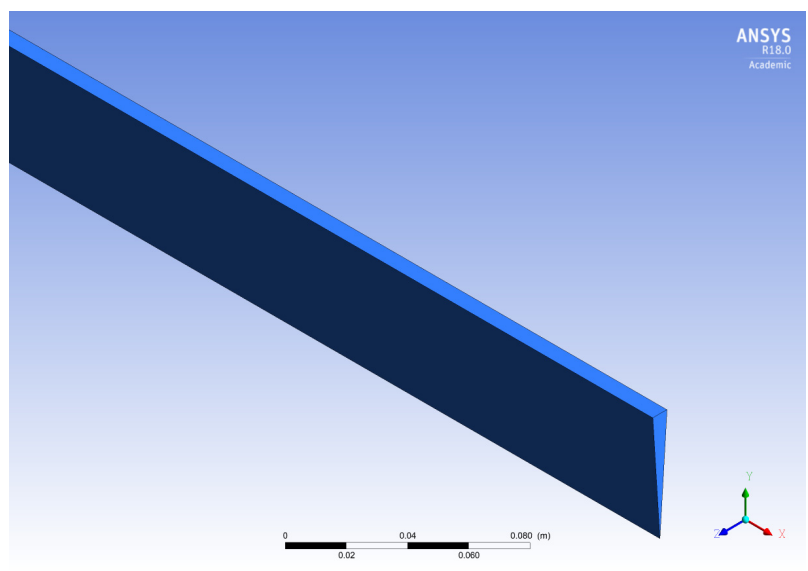

Fig.4. 3D view of the 2D flow domain in CFD-Post

\section{Results}

First of all, the four different viscous flow models are compared in respect of x-velocity, or in other words, streamwise velocity component, wall $\mathrm{y}+$ value, turbulence intensity and turbulence viscosity at $\mathrm{Re}=10,000$ in Figure 5. The velocity component in the graphics is denoted with " $\mathrm{u}$ " and its unit is " $\mathrm{m} / \mathrm{s}$ ". The pipe length or pipe axis is denoted with " $x$ " axis and pipe radius is indicated with " $y$ " axis. Velocity profiles are important since there are information in the literature about velocity profiles versus Re number, characteristic length and distance from the pipe axis. Therefore it is relatively easier to evaluate and assess the obtained results from the calculations. The change of " $u$ " along the " $x$ " axis gives an idea about the hydrodynamic development of the flow. These profiles have steeper changes starting from " $x "=0$ and then their change becomes smoother after some distance. It is expected that the profiles have a zero change at the far end of the pipe after the flow is developed. The profile at the pipe axis is important here because it indicates the velocity magnitude that starts in the inviscid core flow region and continues towards to the developed flow region. So it is easier to evaluate the development of the flow by examining this profile. On the other hand, the profile very close to the wall gives an idea of the development of the boundary layer. 


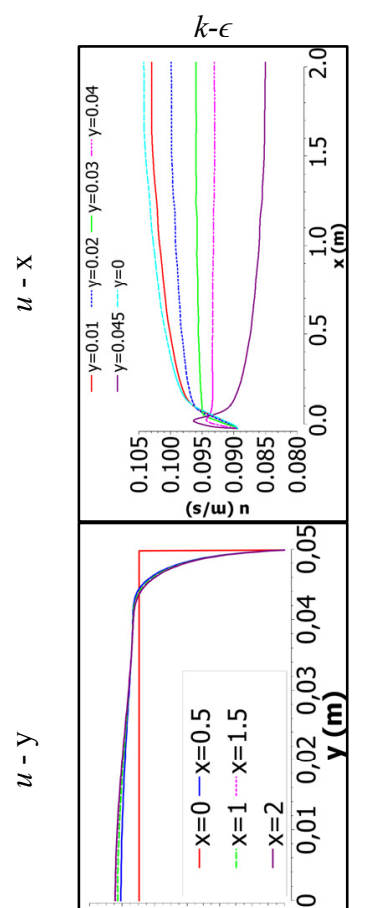

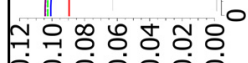

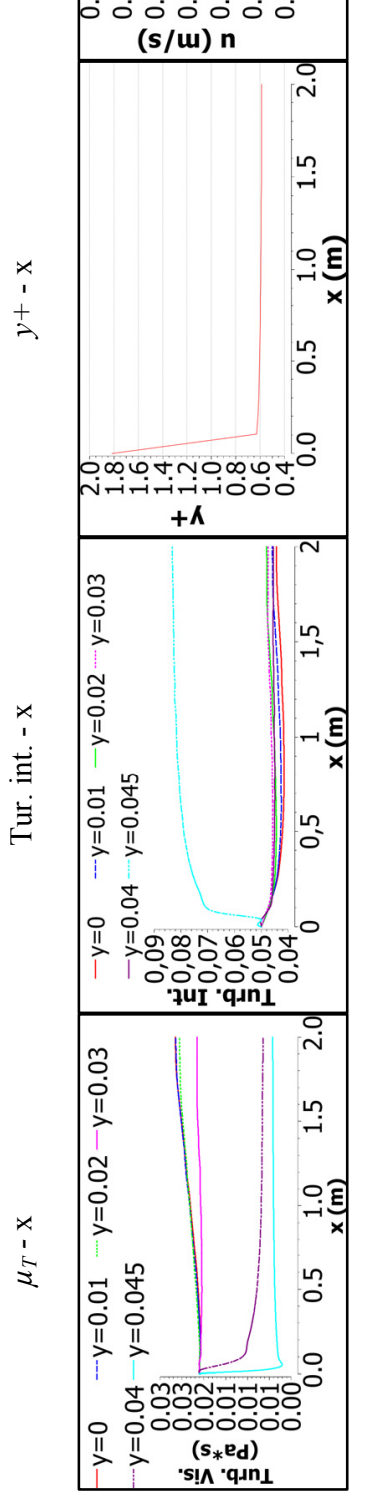

$k-\omega$

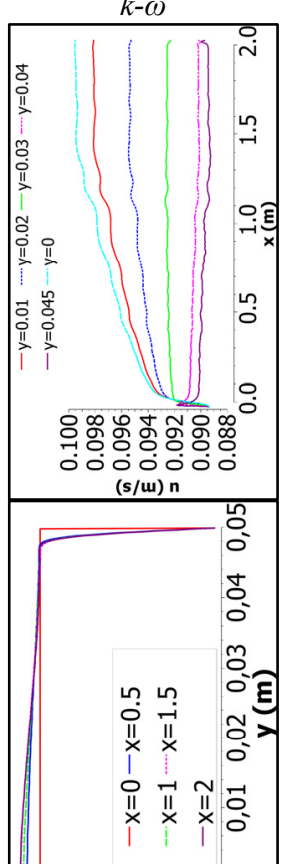

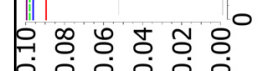

$(\mathbf{s} / \mathbf{m}) \mathbf{n}$
0

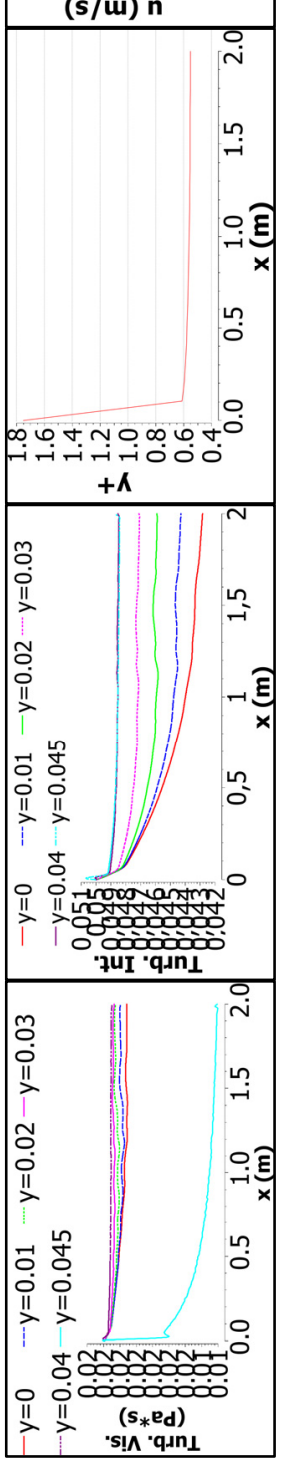

Spalart-Allmaras

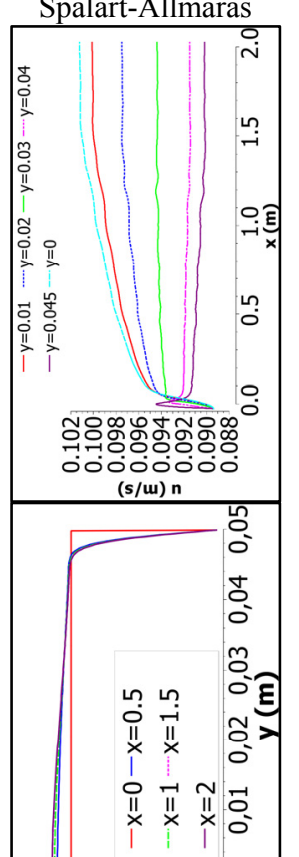

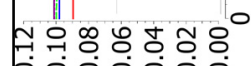

(s/u) n

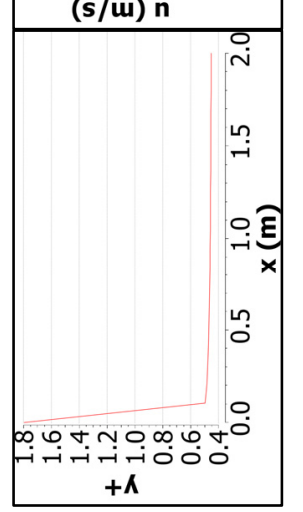

$\frac{0}{0}$
$\frac{\pi}{7}$
$\frac{\pi}{2}$
0
0

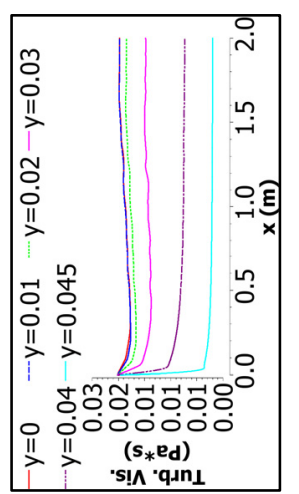

Viscous Laminar

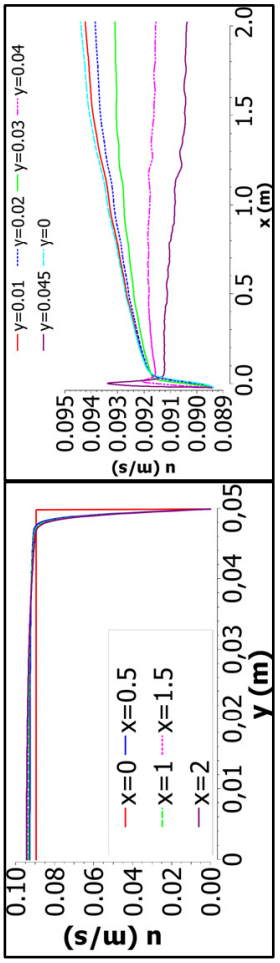

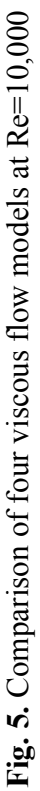

$\frac{j}{2}$

$\frac{0}{0}$
$\frac{\pi}{\pi}$
$z$
$z$
0
$z$ 
At the first glance to Fig. 5, $k$ - $\epsilon$ does not yield too distinguished profiles comparing to the remaining models. The range in velocity magnitude considering all profiles is the least for viscous laminar (VL) model while $k-\omega$ and Spalart-Allmaras (S-A) model follow it respectively. $k-\epsilon$ profiles here have the widest range indicating that this model distinguish more from the VL for $\operatorname{Re}=10,000$. Actually the models other than $k-\epsilon$ imply a higher Re number comparing to profiles of $k-\epsilon$ or in other words vice versa. Turbulent flow develops faster than laminar flow due to the presence of turbulent structures that have much more impact on the flow than the molecular viscosity. The boundary layer rises and is restricted because of the turbulent momentum transfer. In the absence of such momentum transfer, the boundary layer of the laminar flow rises slowly with length towards the center. Since more length is needed for the laminar flow development, the initial part of the developing laminar flow looks like a turbulent one. This is the case here because the length of the pipe is $2 \mathrm{~m}$ and it is well known that practically development length is $10 D_{h}$ for turbulent flows, meaning that $1 \mathrm{~m}$ in the present case. $50 \mathrm{~m}$ is needed for the laminar developed flow. It is interesting to see that the velocity profiles for " $\mathrm{u}$ " is similar that of VL model and current turbulence models for the turbulence entrance region. One can propose that physical phenomena that are related to " $u$ " component can benefit from the ease of the VL model for the turbulence entrance region.

The $k-\epsilon$ developed a thicker boundary layer before remaining models, yet its velocity profile changes smoothly after a distance more close to the $1 \mathrm{~m}$ length. This statement is justified by looking at the " $\mathrm{y} "=0.045$ profile (purple colored). It can be inferred that some of the flow energy is converted into turbulence and hence velocity profile develops faster for $k-\epsilon$ than remaining models. $u$-y profiles show identical inlet velocity profiles for four models. At the outlet, however, VL velocity profile shows that only a big portion has the same velocity magnitude and the maximum magnitude deviates only about $0.003 \mathrm{~m} / \mathrm{s}$ from the initial velocity magnitude while this value is between $0.003-0.005$ for SA, 0.004-0.006 for $k-\omega$ and 0.007-0.01 for $k-\epsilon$.

Wall treatment in numerical investigation considers $\mathrm{y}+$ dimensionless length. Since VL doesn't give this information, only wall $\mathrm{y}+$ values of remaining turbulence models along the pipe length are given in Fig.5. This value is calculated for the cell next to the wall. $\mathrm{y}+$ is desired about 1 for $k$ - $\epsilon$ and below 1 for $k$ - $\omega$ according to the literature. Fig. 5 shows that wall $\mathrm{y}+$ is about 0.6 for $k-\epsilon$ and $k-\omega$. For lower mesh element numbers, $\mathrm{y}^{+}$gets higher values. Although the turbulent flow can be modeled, there is a region close to the wall where viscous effects become stronger and flow behaves like laminar. The molecular viscosity here plays the major role. This region is characterized by $y+$ value which is a dimensionless distance. It is defined as " $y$ " times the friction velocity over kinematic viscosity. $\mathrm{y}+$ between 0 6 indicates viscous sub-layer where $\mathrm{u}+$ is equal to $\mathrm{y}^{+}$. 630 indicate buffer zone and $30-500$ is the log-law zone. In the viscous sub-layer, the fluctuating velocity components diminish and shear stress is only due to the viscosity. The wall functions to be used are determined by looking to $\mathrm{y}+$. Also development of $\mathrm{y}+$ along the perpendicular axis gives information about the development of the boundary layer. Considering above mentioned facts, wall $\mathrm{y}+$ is expected between 0-6.

More turbulent viscosity values are calculated with $k$ $\epsilon$ than the remaining models which means fluctuating velocity components have higher magnitudes over the flow domain. This should be due to the approach in which turbulent viscosity is calculated. Again $k-\epsilon$ is found favorable here because it reveals more information about the turbulence comparing to other models.

Turbulence intensity was estimated as $5 \%$ at the inlet for the initial conditions of the simulation. $k-\epsilon$ yields turbulence intensity values greater than $5 \%$ along the pipe length while $k-\omega$ yields results implying that turbulence diminishes with length and becomes weaker. Still, it is understood that a big portion of the flow field has turbulence intensity value about $5 \%$ in $k-\epsilon$ and $4 \%$ in $k-\omega$. Near the wall, turbulence intensity has values as high as $15 \%$ for $k-\epsilon$ and $6 \%$ for $k-\omega$.

Figure 6 is given for comparison of different Reynolds numbers considering that $k-\epsilon$ is the turbulence model. Looking to the Fig. 6, only $\mathrm{Re}=50,000$ shows a different $u-x$ profile near the wall, indicating that boundary layer becomes narrower as expected. u-y velocity profiles are similar for $\mathrm{Re}=50,000$ and 10,000 while they become more parabolic for transition region of $\mathrm{Re}$ and laminar Re. The maximum u velocity values for $\mathrm{Re}=50,000,10,000,5,000$ and 1,000 respectively are $0.475 \mathrm{~m} / \mathrm{s}, 0.1 \mathrm{~m} / \mathrm{s}, 0.05 \mathrm{~m} / \mathrm{s}$ and $0.011 \mathrm{~m} / \mathrm{s}$. The maximum $u$ velocity values also indicate that the maximum values increase more as it goes further into the laminar region since we can compare 10 fold decreased $\mathrm{Re}$ number maximum $\mathrm{u}$ velocity value with the $\mathrm{u}$ velocity value of the Re number by omitting one " 0 " after the decimal. The wall $\mathrm{y}+$ become closer to 6 value for $\mathrm{Re}=50,000 . \mathrm{y}+$ values develop after $0.1 \mathrm{~m} \times$ length. Turbulence intensity is about 3.5 fold of $\operatorname{Re}=10,000$ for $\mathrm{Re}=50,000$ which implies that the increase of intensity is not linear comparing to the increase in Re. Turbulence intensity changes between $27 \%$ and $20 \%$ for $\mathrm{Re}=50,000$, $8 \%$ and $5 \%$ for $\mathrm{Re}=10,000,5 \%$ and $2 \%$ for $\mathrm{Re}=5,000$ and $2 \%$ and $0.5 \%$ for $R e=1,000$. Turbulent viscosity tends to get a common value for further pipe length and $\mathrm{Re}=10,000$ and below. However $\mathrm{Re}=50,000$ is different in this respect since pipe radius values away from the wall (starting from $\mathrm{y}=0.03$ and goes towards $\mathrm{y}=0.01$ ) yield turbulent viscosity values diverge from turbulent viscosity values of pipe radiuses close to wall. These results also imply that turbulence magnitude through the flow domain is nearly homogenous or in other words doesn't get too much different values. Looking to the ranges of the results in the $2 \mathrm{D}$ plots, turbulence is calculated for $\mathrm{Re}=1,000$ however the calculated results have relatively small magnitudes. Therefore the model can also be thought for the solution of the laminar flows. 

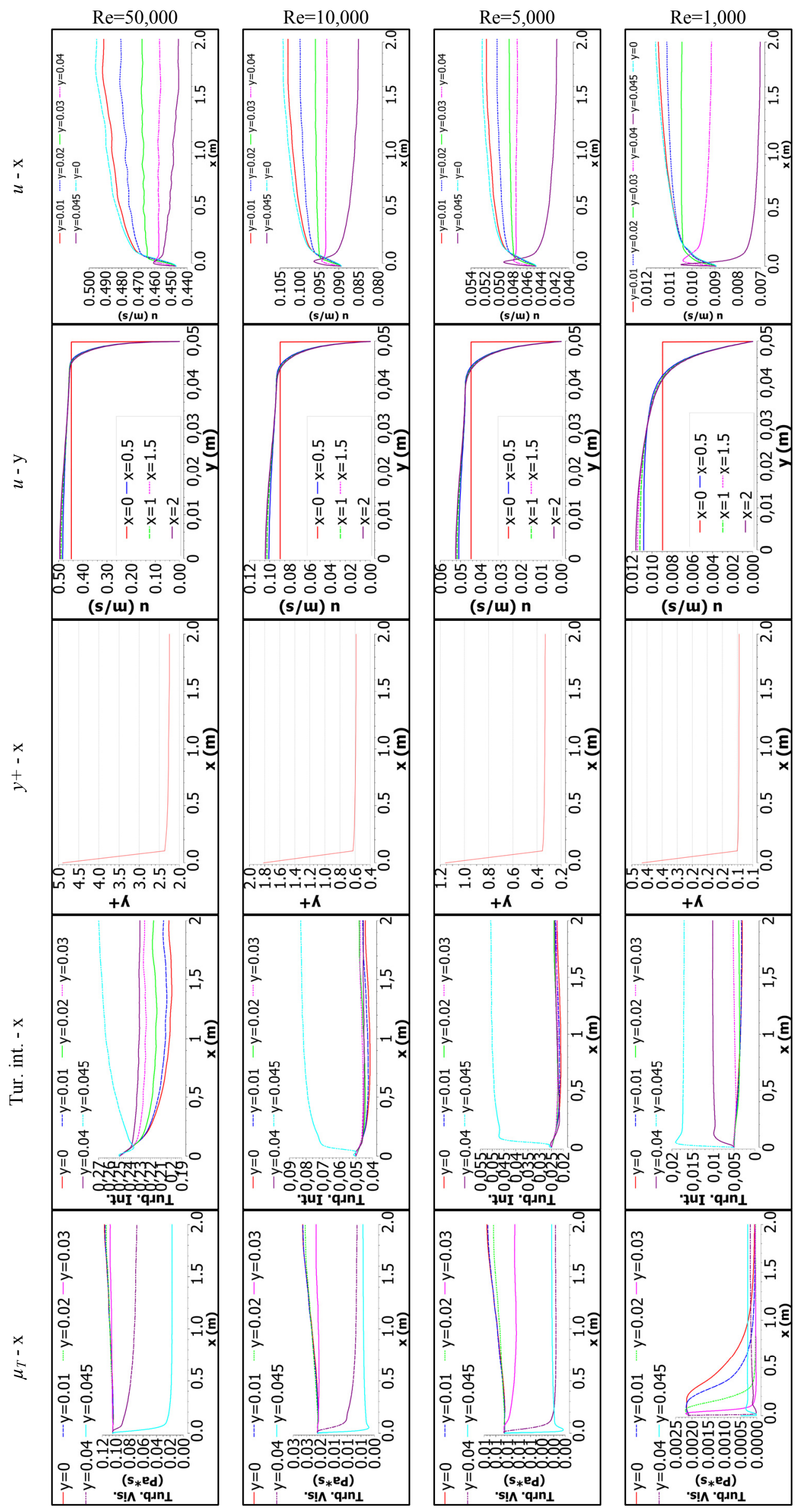

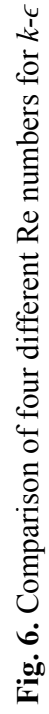




\section{Conclusions}

This work aims to declare an ongoing $\mathrm{PhD}$ work and its foundation. The motivation of the work is tried to be explained by a literature timeline. The governing equations in $2 \mathrm{D}$ cylindrical coordinates are given. Then a preliminary computational fluid dynamics analysis is reported in this paper for a similar turbulent pipe flow case. Ansys CFD commercial software is utilized and four different viscous flow models were compared. Finally $k-\epsilon$ turbulence model results for four different Reynolds numbers are presented. Following remarks can be drawn from the text.

- The $u-y$ velocity profiles show that the turbulent flow develops after 10 hydraulic diameters as an experimental justification of the results.

-The increase in mesh element number yield unfavorable results after a certain level and it is thought that this is due to the very small lengths leading to numerical errors. The information of free stream turbulence is also decreasing with increasing mesh element number.

- $k$ - $\epsilon$ yields more information about the turbulence considering the whole of the flow domain.

- $k-\omega$ can be preferred if near wall region is specifically important.

- The models don't make a significant difference in time cost of the simulation.

- Mesh (grid) elements between 20,000 and 80,000 elements are found favorable for a similar case for further analyses.

Although the method and the findings are related with CFD, experimental results and facts both establish a basis for justification and provide data for developing modeling. For instance, $u-y$ velocity profiles of the viscous flow models of the present study is compared to the practical flow development length of turbulent flows in reality which was experimentally obtained in the past. Also it is known that the CFD is used for phenomena that are hard to be measured but in the light of experimentally obtained experimental boundary conditions.

Deeper parametrical analyses of the computational events related to the results will be done in the future for a deeper understanding of the turbulence modeling. Also it is planned to establish a laboratory setup that can enable justification of CFD results by hotwire measurements, led type PIV and thermal camera applications.

\section{Acknowledgment}

The academic license of the software used in this work is belonged to Selcuk Univertsity. Authors also would like to acknowledge the financial support of Selcuk University Scientific Research Projects Coordination Unit for the conference participation (project no: 17701387).

\section{References}

1. S. Bilir, Selcuk University, Natural and Applied Sciences Ins., PhD Thesis, Konya, TURKEY (1988)

2. S. Bilir, Int. J. Heat Mass Transfer., 38, 9 (1995)

3. S. Bilir, International Journal of Heat and Mass Transfer, 45 (2002)

4. S. Bilir, A. Ates, International Journal of Heat and Mass Transfer, 46 (2003)

5. A. Ates, Selcuk University, Natural and Applied Sciences Ins., PhD Thesis, Konya, TURKEY (1998)

6. A. Ates, S. Darici, S. Bilir, International Journal of Heat and Mass Transfer, 53 (2010)

7. S. Darici, Selcuk University, Natural and Applied Sciences Ins., PhD Thesis, Konya, TURKEY (2004)

8. S. Darici, A. Ates, S. Bilir, J. Fac.Eng.Arch. Selcuk Univ., 20, 3 (2005)

9. S. Darici, A. Ates, S. Bilir, Journal of SelcukTechnic, 7, 2 (2008)

10. S. Darici, S. Bilir, A. Ates, International Journal of Heat and Mass Transfer, 84 (2015)

11. A.H. Altun, Selcuk University, Natural and Applied Sciences Ins., PhD Thesis, Konya, TURKEY (2013)

12. A.H. Altun, S. Bilir, A. Ates, International Journal of Heat and Mass Transfer, 92 (2016)

13. H. Demirpolat, Selcuk University, Natural and Applied Sciences Ins., PhD Thesis, Konya, TURKEY (2015)

14. S.U. Atmaca, Selcuk University, Natural and Applied Sciences Ins., PhD Thesis, Konya, TURKEY (2013)

15. S. Sen, Selcuk University, Natural and Applied Sciences Ins., PhD Thesis, Konya, TURKEY (2016)

16. S. Sen, S. Darici, Applied Thermal Engineering, 111 (2017)

17. N. Kasagi, A. Kuroda, M. Hirata, J. Heat Transfer, 111, 2 (1989)

18. Y. Cao, A. Faghri, J. Heat Transfer, 114, 4 (1992)

19. W. Jaeger, Nuclear Engineering and Design, 319 (2017)

20. I. Tiselj, R. Bergant, B. Mavko, I. Bajsic, G. Hetsroni, J. Heat Transfer, 123 (2001)

21. I. Tiselj, L. Cizelj, Nuclear Engineering and Design, (2012) DOI: 10.1016/j.nucengdes.2012.08.008

22. D.A. Anderson, J.C. Tannehill, R.H. Pletcher, Computational Fluid Mechanics and Heat Transfer, McGraw-Hill (1984)

23. W.P. Jones, B.E. Launder, Int. J. Heat Mass Transfer, 15 (1972)

24. B.E. Launder, D.B. Spalding, Comput. Meth. Appl. Mech. Engg, 3 (1974) 